\title{
Impact de la vidange sur le développement des populations zooplanctoniques dans une retenue des Pyrénées
}

\author{
J. Rey 1
}

Mots clés : zooplancton, vidange, réservoir, Pyrénées.

Les pertes de zooplancton dans l'exutoire du lac d'Orédon durant la période de vidange (21 juillet - 31 octobre) ont été évaluées à 3,1 tonnes PF.

La nature et la quantité du zooplancton exporté sont liées au comportement migratoire des organismes pélagiques, à l'amplitude de leur déplacement vertical et à la variation de hauteur de la colonne d'eau.

Les ponctions successives exercées par la vidange durant la phase de croissance des especes amoindrissent le peuplement zooplanctonique et la productivité du réservoir. L'incidence des pertes à l'exutoire relativement compensée chez. les espèces à court temps de générat ion (Daphnia longispina) se traduit à long terme par l'élimination de formes à long cycle de développement (Mixodiapıomus laciniaus).

Impact of emptying on the development of zooplankton populations in a Pyrenean reservoir

Keywords : zooplankton, emptying, reservoir, Pyrenees.

The loss of zooplankton in the outflow of Lake Oredon during a period of emptying (21 July to 31 October) has been estimated as 3.1 tonnes (wet weight).

The nature and quantity of exported zooplankton were affected by the migratory behaviour of the pelagic organisms, by the amplitude of their vertical displacement and by the variation in the height of the water column.

The successive pulses caused by the emptying during the growth phase of the species reduces the zooplankton population and the productivity of the reservoir. The losses at the outflow are partially compensated by species with short generation times (Daphnia longispina), but in the long term lead to the loss of species with a long development cycle (Mixodiaptomus laciniatus).

\section{Introduction}

La dérive de zooplancton hors des lacs et des réservoirs a fait l'objet de nombreuses publications parmi lesquelles, ces dernières décennies, celles de : Eliott \& Corlett (1972), Neveu \& Echaubard (1975), Ward (1975), Armitage \& Capper (1976). L'objectif de ces travaux est d'évaluer la quantité de matière organique rejetée hors des écosystèmes et d'étudier son devenir au cours du transport aval et son influence sur la st ructure des communautés benthiques. Généralement, peu de données sont foumies

1. Laboratoire d'Hydrobiologie, UA C.N.R.S. 695, Université Paul Sabatier, 118, route de Narbonne. 3 t062 Toulouse Cédex. France. sur les communautés zooplanctoniquęs des lacs dont ces organismes sont issus.

Dans le cadre d'une étude de la dynamique de populations et de la production du zooplancton de quelques lacs d'altitude des Pyrénées, les pertes de zooplancton ont été étudiées à la sortie du lac de barrage d'Orédon. Le but de ces observations, menées parallèlement à celle des populations zooplanctoniques dans la retenue, était d'estimer la nature et la quantité de zooplancton rejeté dans l'exutoire et l'incidence de cet export sur la structure de la communauté zooplanctonique dans le réservoir.

Une partie des résultats a été publiée an térieurement (Rey 1984). Des données complémentaires sont présentées dans cet article. 


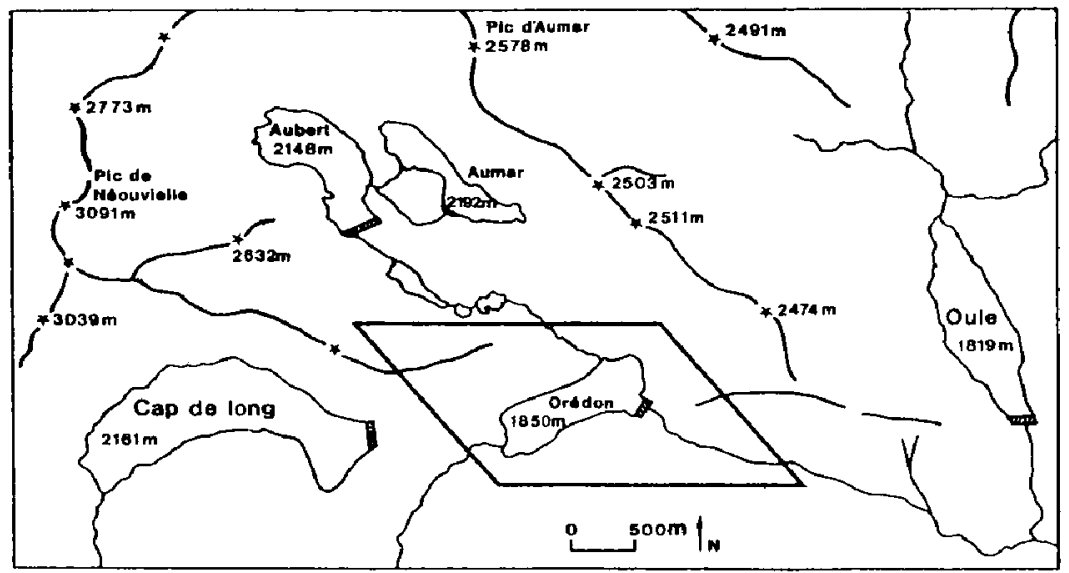

Fig. 1. Localisation du lac d'Orédon dans le complexe lacustre hydróélectrique du Néouvielle (Pyrénées Centrales).

\section{Milieu et méthodes}

Le lac d'Orédon ( $1849,40 \mathrm{~m}$ à sa cote maximale) appartient au complexe hydro-élect rique du Néouvielle (fig. 1). Ses principales caractéristiques morphométriques sont les suivantes:

Superficie (ha)

Volume $\left(10^{6} \mathrm{~m}^{3}\right)$

Profondeur max. (m) 59

Profondeur moy. (m)

40

Profondeur de la prise d'eau (m) $\quad-24,3$

La finalité de ce réservoir est d'assurer les besoins de l'irrigation en plaine en été-automne et l'alimentation des centrales hydro-électriques en hiver. Le remplissage s'effectue à la fonte des neiges et la vidange a généralement lieu en hiver. Cependant une vidange partielle ou même totale peut survenir en été selon la demande de l'agriculture. En 1976, les opérations de vidange ont été réalisées du 26 juillet au 31 octobre.

L'étude du matériel zooplanctonique est basée sur 9 séries d'échantillonnages effectuées tous les 7 à 10 jours, du début à la fin de la période de vidange.

De plus, deux séries d'observations sur un cycle de 24 heures, réalisées en période de hautes eaux
(5-6 VIII) et de basses eaux (30.IX - 1.X), ont été menées simul tanément à l'exutoire et dans la retenue, afin d'analyser la relation entre la quantité des organismes exportés et leur distribution verticale dans le réservoir.

Les prélèvements ont été effectués à la sortie des vannes du barrage au moyen d'un filet de ClarkeBumpus (vide de maille $175 \mu$ ) maintenu dans le déversoir pendant trois minutes à différentes heures de la journée.

Les comptages ont été effectués à partir de six sous-échantillons au minimum, réalisés avec une pipet te Hydrobios calibrée à $5 \mathrm{cc}$. Pour chaque série de sous-échantillons, le type de distribution a été vérifié par le test $d u x^{2}$. Dans tous les cas, les valeurs du $x^{2}$ sont en conformité avec une loi de Poisson au seuil $\mathbf{P} 0.05$.

Le nombre d'organismes zooplanctoniques exportés lors de chaque relevé a été évalué à partir de l'enregistrement de la quantité d'eau filtrée par le filet et des données de débit fournies par EDF.

Les valeurs de biomasse ont été obtenues à partir des données de poids sec évaluees pour les 


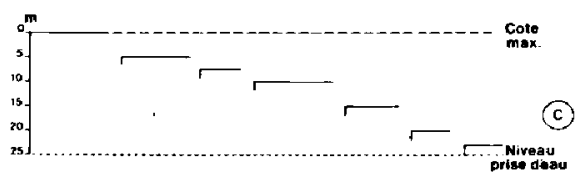

\section{Résultats}

Les figures 2 à 4 rappellent les caractéristiques de l'export de matériel zooplanctonique dans l'exutoire du lac d'Orédon.

a. - Les pertes de zooplancton (fig. 2) sont maximales fin juillet, période ou sont enregistrés les plus forts lâchers de barrage (soit 3 milliards d'individus représentant une biomasse de $165 \mathrm{~kg}$ PF pour 24 heures, pour un volume de $475000 \mathrm{~m}^{3} \mathrm{~d}^{\text {eeau écou- }}$ lés à l'émissaire). Elles diminuent ensuite régulièrement jusqu'à la fin des observations. La quantité de zooplancton exporté durant la période de vidange (26 juillet - 31 octobre) a été estimée à 3,1 tonnes PF.

b. - L'élément dominant les organismes rejetés hors de la retenue (Tableau I) est Daphnia longispina qui constitue à elle seule 50 à $85 \%$ de la biomasse exportée. Cyclops strenuus est exporté en quantité moindre. Kellicottia longispina représente une fraction négligeable $(0,02$ à $0,07 \%)$ de l'export total.

c. - L'export de zooplancton dans l'exutoire de ce réservoir résulte de l'interférence des organismes au cours de leurs déplacements verticaux avec les couches d'eaux soumises à départ par les vannes.

La majeure partie de cet export s'effectue en phase diurne en période de hautes eaux, en phase nocturne en période de basses eaux (fig. 3).

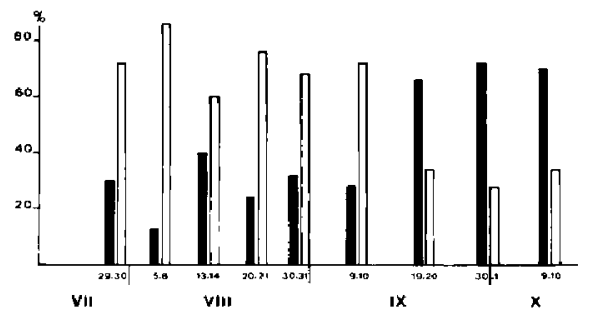

Fig. 3. Valeurs relatives de la biomasse zooplanctonique exportée en phase diurne (blanc) et en phase nocturne (noir).

L'inversion du processus s'explique par l'analyse sur un cycle de 24 heures de la répartition verticale de Daphnia longispina et des pertes en registrées simul tanément pour cet te espèce, en période de hautes eaux (5-6 VIII) et en période de basses eaux (30.IX - I.X). 
Tableau I. Export de zooplanctón à la sortie des vannes du barrage d'Orédon durant la période de vidange. Composition centésimale du peuplement et de la biomasse aux différentes dates d'observations. Nombre total d'individus et poids frais exportés par 24 heures avec limites de confiance à P 0,05.

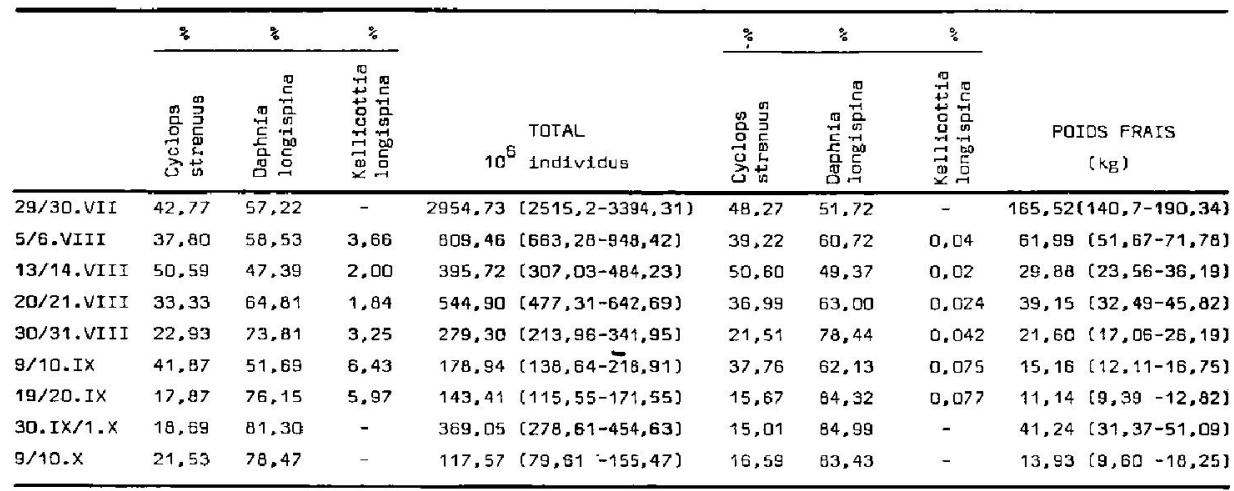

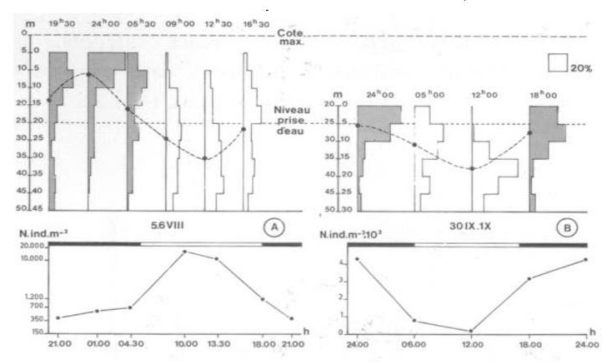

Fig. 4. Pertes à l'exutoire et répartition verticale de Daph. nia longispina le 5-6 VIII (A) et les 30.IX - 1.X (B). Les courbes en pointillés indiquent la limite des $50 \%$ de la population.

Dans les 2 séries d'observations (fig. 4), Daphia montre le comportement migratoire typique chez les organismes planctoniques : concentration nocturne en surface, enfoncement diume.

- Les 5-6 VIII (cote du réservoir: $-5 \mathrm{~m}$ ), la population en majorité abritée dans les 10 premiers mètres durant les heures de nuit, se trouve exposée au passage de la couche $-25 \mathrm{~m}$ lors des séquences diumes d'enfoncement et de remontée vers la surface. Les pertes consécutives représentent $85 \%$ des pertes enregistrées pour les 24 heures (fig. 4a).

- Les 30.IX - I.X (niveau du réservoir proche de celui de la prise d'eau), la population, en profondeur durant le jour, se trouve exposée lors de la venue en surface des individus. La majorité des pertes subies par l'espèce se produit en phase nocturne (fig. 4b).

Un examen plus approfondi du matériel rejeté dans l'exutoire a en outre révélé que l'export affecte différemment les organismes, selon qu'ils présentent une plus ou moins grande amplitude de leur déplacement vertical.

Ainsi l'analyse par catégorie d'individus $\sigma$ et $\%$, des Daphnia dénombrées à la sortie des vannes du barrage montre, tout au long de la période d'observation, une proportion de $\sigma^{\circ}$ presque toujours supérieure à celle observée simul tanément dans les relevés de la retenue (fig. 5).

L'étude de la répartition verticale au cours d'un cycle de $24 \mathrm{~h}$ effectuée séparément pour chaque catégorie d'individus (fig. 6), révèle que la population ơ se retrouve en quasi totalité dans les couches 


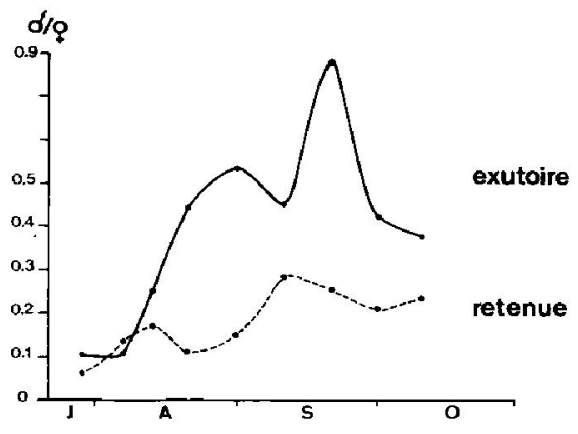

Fig. 5. Evolution comparée du rapport $\sigma / q$ dans le lac d'Orédon et dans son exutoire. de surface durant les heures de nuit, et que le déplacement vertical au cours du nycthemere est, pour la moyenne de la population, de moins grande amplitude que celui des femelles.

Ce comportement est à l'origine des pertes conséquentes d'individus $\sigma$ enregistrées dans l'exutoire tout au long de la période de vidange.

L'ensemble du processus est illustré par la figure 7 .

- En période de hautes eaux (observations des 5-6 et 13-14 août), la fraction or de la population évoluant dans les couches superficielles en phase nocturne, se trouve exposée durant un temps réduit du cycle nycthéméral : les pertes en individus sont relativement limitées.

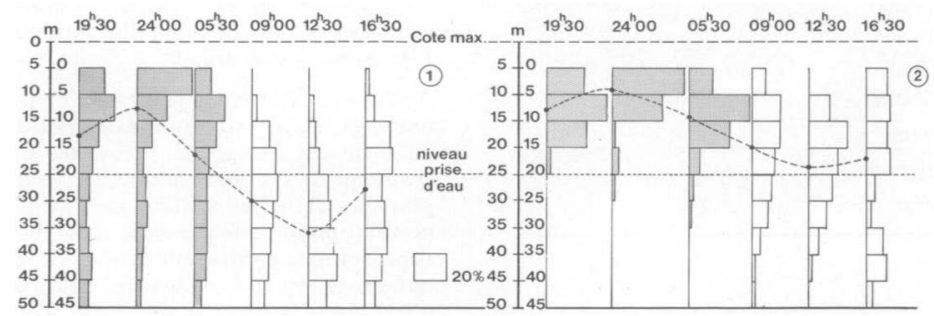

Fig. 6. Répartition verticale au cours d'un cycle de 24 heures des individus femelles (1) et mâles (2) de Daphnia longispina dans le lac d'Orédon. En tireté : limite des $\mathbf{5 0}$ ơ de la population.
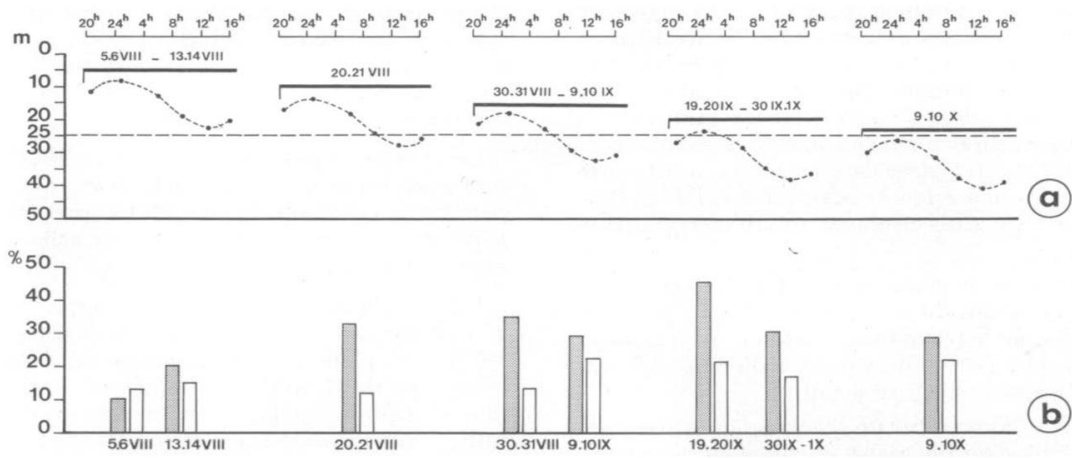

Fig. 7. a : schéma d'interprétation. Répartition verticale des mâles de Daphnia longispina dans la retenue au cours de la période de vidange. Les courbes en tirets indiquent la limite des $50 \%$ de la population.

$\mathrm{b}$ : abondance relative comparée des mâles de Daphnia longispina dans l'exutoire (pointillés) et dans la retenue (blanc) 
Tableau II. Pertes quotidiennes à l'exutoire du lac d'Orédon des individus $\alpha$ et $\vartheta$ de Daphnia longispina en pourcentage des populations mâle et femeille présentes dans la retenue.

(Ces populations ont été évaluées en intégrant par strates de $5 \mathrm{~m}$ de la surface au fond, pour chaque catégorie d'individus, le produit du nombre moyen d'individus présents dans chaque strate sous une surface de $1 \mathrm{~m}^{2}$ de section, par le volume de la strate considérée. Les volumes ont été calculés d'après la courbe hypsographique du réservoir (Uzabiaga 1978).

\begin{tabular}{lll}
\hline & \multicolumn{1}{c}{$\delta$} & $q$ \\
\cline { 2 - 3 } 30 juillet & 4 & 4,7 \\
5/6 août & 0,7 & 1,2 \\
13/14 août & 0,5 & 0.4 \\
20/21 août & 4 & 1,1 \\
30/31 ao0t & 2,3 & 0,7 \\
9/10 septembre & 0.3 & 0.3 \\
19/20 septembre & 1,5 & 0,4 \\
30 sept./1 oct. & 5,2 & 2,8 \\
g/10 ootabre & 1,7 & 1,1 \\
\hline
\end{tabular}

- Les baisses de niveau se poursuivant, cette même catégorie d'individus est appelée à séjourner durant une portion plus importante du nycthérnère dans les couches soumises à départ par les vannes. Il en résulte un nombre d'autant plus grand d'individus entraînés dans l'exu toire cobservations de fin août à fin septembre, période durant laquelle la proportion de $\sigma^{\circ}$ enregistrée dans les relevés à l'exu toire se révèle jusqu'à 3 fois supérieure à celle enregistrée dans les relevés effectués simultanément dans la retenue).

- En fin de période de vidange (9-10 octobre), lorsque le niveau du réservoir se rapproche de sa cote minimale, le phénomène s'at ténue mais la population reste encore soumise de façon non négligeable à l'action de la prise d'eau.

La figure 7a constitue un schéma d'interprétation de l'ensemble du phénomène. Elle a été réalisée à partir des observations effectuées les 5-6 août (fig6 ), en supposant pour les individus un comportement migratoire identique lors de chaque relevé.

\section{Discussion}

La quantité de matière organique rejetée dans l'exu toire du lac d'Orédon, soit 3,1 tonnes PF pour la période de vidange (21 juillet - 31 octobre 1976), représente une perte conséquente pour les populations zooplanctoniques de la retenue. Dans 2 réservoirs alpins, l'Amersee et le Weisssee, Pechlaner (1964) évalue la décharge de zooplancton à 1,4 et 25 tonnes respectivement.

Les pertes de Daphnia à l'exutoire d'Orédon représentent une part importante de la mortalité de l'espece.

Le calcul des équations de diminution des nombres, soit

$$
\begin{array}{ll}
\mathrm{N}_{\mathrm{t}}=\text { No.e } & -0,00529 \mathrm{t} \\
& -0,01446 \mathrm{t} \text { pour les } \sigma \\
\mathrm{N}_{\mathrm{t}}=\text { No.e } & \text { pour les } \%
\end{array}
$$

indique des taux de mortalité journaliers de 0,53 et $1,45 \%$ respectivement.

Si l'on examine les pertes à l'exutoire des Daphnia or et $q$ en pourcentage des populations de chacune de ces catégories présentes dans la retenue (Tableau II), on observe que les pertes enregistrées pour les femelles varient de $0,4 \%$ à $2,8 \%$ de la population femelle présente dans le réservoir. Elles représentent en moyenne, 60 à $70 \%$ du taux journalier de mortalité évalué pour cette catégorie d'individus.

Les pertes enregistrées pour les mâles varient de 0,3 à $5,2 \%$ de la population mâle peuplant la retenue. Elles représentent la quasi totalité de la disparition des individus. Par ailleurs, elles expliquent le sex-rat io relativement faible observé dans ce réservoir, comparativement aux valeurs très largement supérieures enregistrées pour la même espèce dans 2 retenues voisines (Rey 1988).

Les pertes importantes observées le 30 juillet tant pour les $\sigma$ que pour les $Q(4 \%$ et $4,7 \%$ respectivement des populations $\sigma$ et $\%$ présentes dans le réservoir) correspondent aux forts lâchers du barrage à cette date.

L'étude du zooplancton en dérive dans le déversoir du lac naturel de Port-Bielh (Rey \& Capblancq 1975), avait montré, en pourcentage des populations pélagiques de Mixodiaptomus laciniatus et de Daphnia longispina, une perte journalière de $0,28 \%$ en juillet pour le Diaptomide et de $0,014 \%$ en septembre pour le Cladocère.

Les pertes de Daphnia dans l'exutoire du lac d'Orédon le 30 juillet, se révèlent plus de 600 fois 
supérieures. Cette disparité souligne l'impact exercé par la vidange sur les populations zooplanctoniques de la retenue. Elle explique également la disparition de Mixadiaptomus laciniatus qui peuplait autrefois la zone pélagique du lac d'Orédon (Lagarrigue 1949). Présent dans le plancton du réservoir jusqu'aux alentours des années 60, ce Copépode avait totalement disparu une décennie plus tard.

Si l'on transpose les pertes considérables de Daph. nia enregistrées pour ce réservoir à la population de Mixodiaptomus laciniatus, il apparait que les ponctions successives exercées par les opérations de vidange sur la population du diaptomide ont entraîné sa disparition.

Des modifications survenues ces dernières décennies dans l'exploitation des réservoirs du complexe hydro-électrique ont probablement joué un rôle déterminant. Jusqu'en 1954 en effet, le lac d'Orédon recevait les eaux d'un réservoir amont : le lac de Cap de Long (cf. fig. 1). A partir de 1954, les eaux de ce réservoir ont été dérivées sur une autre vallée. Dès lors, ne bénéficiant plus d'apports compensateurs, le lac d'Orédon a été soumis à de plus importantes variations de sa masse d'eau de façon plus ou moins précoce, selon les années hydrologiques.

La vidange estivale étant concomittante de la phase de croissance des espèces (Rey 1988), pour Mixodiaptomus laciniatus, forme à long temps de génération, la perte de stades naupliens et de copépodites au cours de l'été, en réduisant le nombre d'adultes produits dans ce réservoir, a peu à peu entraîné une diminution de son potentiel de peuple. ment. Progressivement affaiblie et toute possibilité d'ensemencement à partir de Cap de Long (peuplé par le même diaptomide) supprimée, l'espèce a fini par disparaitre.

L'importance de la décharge dans le contrôle du développement du plancton des lacs a été diversement notée (Ruttner 1956, Axelson 1961, Rodhe 1964, Buikema \& Loeffelman 1978). Brook \& Woodward (1956) soulignent le fait que des taux élevés de renouvellement de l'eau peuvent entraîner des différences non seulement quantitatives mais qualitatives dans le plancton lacustre.

Dans le lac d'Orédon les vidanges estivales, outre qu'elles se traduisent par une forte diminution de la productivité du milieu (Rey, op.cit.), ont entraîné une restructuration de la communauté pélagique des Crustacés désormais réduite à Duphnia longispina et Cyclops strenuts. Ces deux espèces se névèlent moins affectées par le phénomène en raison du cycle de développement comparativement plus rapide pour le Cladocère, et du mode de vie pseudobenthique du Cyclopide (Rey \& Dupin 1973).

Travaux cilds

Armilage (P.D.) \& Capper (M.H.). 1976. - The numbers, biomass and transport downstream of microcrustaceans and Hydra from Cow Green Reservoir (Upper Teesdale). Fres $h w$ : Biology, $6: 425-432$.

Axelson (J.). 1961, - Zooplankton and impoundment of two lakes in Northern Sweden (Ransaren and Kultsjön). Repr. Inst. Freshw. Res. Drottningholm, 42 : 84-168.

Brook (A.J.) \& Wcoodward (W.B.). 1956. - Some observations on the effects of water inflow and outflow on the plankton of sinal] lakes. J. Anim. Ecol., $25: 22-35$.

Buikema (A.L.) \& Loeffelman (P.H.). 1978. - Effects of pumpback operations on the zooplankton dynamics of Smith Mountain Lake, Virginia Virginia Polytechnic Lnstitute and State University Blacksburg, Virginia. 128 pp-

Buikema (A.L.) \& Loeffelman (P.H.). 1978. - Effects of pumped storage operations on rotifer populations. Verh. Intemat. Verein. Limnol, $20=1597-1603$.

Elliott (J.M.) \& Corlett (J.). 1972. - The ecology of Morecambe Bay. IV. Invertebrate drift into and from the river Leven. $J_{\text {. appl. }}$ Ecol., 9 (1): 195-205.

Lagarrigue (J.) 1949. - Le zooplancton du lac d'Orédon. Bull Soe. Hist. nat. Toulouse, $84(1.2): 45-53$.

Neveu (A.) \& Echaubard (M.). 1975. - La derive estivale des invertébrés aquat iques et terrest res dans un ruisseau du massif central : la Couze Pavin. Ann. Hycirobiol., 6 (1): 1.26 .

Pechlaner (R.). 1964. - Plankton production in natural lakes and hydro-électric water-basins in the alpine region of the austrian Alps. Verh. Intemas. Verein. Limnol., $15: 375-383$.

Rey (J.). 1984. - Export de maté riel zooplanctonique dans l'exutoire d'une retenue des Pyrénées. Verh. Internat. Verein. Lim. nol., $22: 1493-1497$.

Rey (J, ) 1988. - Etude comparée de la dynamique du zooplancton de trois réservoirs d'altitude et d'un lac naturel dans les Pyré nees. Ammls Limnol., 24 (2): 139-160.

Rey (J.) \& Capblancq (J.). 1975. - Dynarnique des populations et production du zooplancton du lac de Port-Bielh (PyrénéesCentrales). Ann's Limnol, 11 (1): 145.

Rey (J.) \& Dupin (B.) 1973. - Ecologie des Crustacés benthiques du lac de Port-Bielh (Pyrénées Centrales). II. Cycles biologiques. Arnls Limnol., 9 (3): 259.271 .

Rhode (W.) 1964. - Effects of impoundment on water chemistry and plankton in Lake Ransaren (Swedish Lappland). Verh. Intermat Vevein. Limnol-1 $15: 437.443$.

Rutuner (F.) 1956. - Einige Beobachtungen über das Verhalten des Planktons in Seeabflüssen. Ost. bot. z, 103: 98-109.

Ruttrer-Kolisko(A.). 1977. - Suggestions for biomass calculation of plankton rotifers. Arch. Hydrobiol. Beih. Ergebn. Limnol., $8: 71 \cdot 76$.

Uzabiaga (G.). 1978. - Morphométrie, physico-chimie et phytoplancton de trois lacs de barrage daltitude des Pyrenées. Thèse de $3^{*}$ cycle, Hydrobiologie. Univ. P. Sabat ier, Toulouse : $111 \mathrm{p}$.

Ward (J.V.). 1975. - Downstream fate of zooplankton from a hypo limnial release mountain reservoir. Verh. Internat. Verein Limnol., $19: 1798 \cdot 1904$ 